\title{
Malaria seroprevalence in blood bank donors from endemic and non-endemic areas of Venezuela
}

\author{
Carmen Elena Contreras ${ }^{1 /}{ }^{+}$, Marcos De Donato ${ }^{2}$, María Ana Rivas ${ }^{1}$, \\ Hectorina Rodulfo' ${ }^{2}$, Robert Mora ${ }^{3}$, María Eulalia Batista', Norka Marcano ${ }^{4}$
}

${ }^{1}$ Instituto de Inmunología, Universidad Central de Venezuela, Caracas, Venezuela ²Laboratorio de Genética Molecular, Instituto de Investigaciones en Biomedicina y Ciencias Aplicadas, Universidad de Oriente, Cumaná, Venezuela ${ }^{3}$ Banco de Sangre, Hospital JG Hernández, Puerto Ayacucho, Venezuela ${ }^{4}$ Banco de Sangre, Hospital Ruiz y Páez, Ciudad Bolívar, Venezuela

In Venezuela, a total of 363,466 malaria cases were reported between 1999-2009. Several states are experiencing malaria epidemics, increasing the risk of vector and possibly transfusion transmission. We investigated the risk of transfusion transmission in blood banks from endemic and non-endemic areas of Venezuela by examining blood donations for evidence of malaria infection. For this, commercial kits were used to detect both malaria-specific antibodies (all species) and malaria antigen (Plasmodium falciparum only) in samples from Venezuelan blood donors $(n=762)$. All samples were further studied by microscopy and polymerase chain reaction (PCR). The antibody results showed that $\mathrm{P}$. falciparum-infected patients had a lower sample/cut-off ratio than Plasmodium vivax-infected patients. Conversely, a higher ratio for antigen was observed among all $\mathrm{P}$. falciparum-infected individuals. Sensitivity and specificity were higher for malarial antigens (100 and 99.8\%) than for antibodies (82.2 and 97.4\%). Antibodypositive donors were observed in Caracas, Ciudad Bolivar, Puerto Ayacucho and Cumaná, with prevalences of 1.02, 1.60, 3.23 and 3.63\%, respectively. No PCR-positive samples were observed among the donors. However, our results show significant levels of seropositivity in blood donors, suggesting that more effective measures are required to ensure that transfusion transmission does not occur.

Key words: seroprevalence - malaria - diagnosis - blood donors

Venezuela and Haiti are the only two American countries that have exhibited increased numbers of malaria cases in the last nine years, falling short of the goals of the Roll Back Malaria Initiative (WHO 2008). In Venezuela, 363,466 cases of malaria were reported between 1999-2009; with annual fluctuations and regional differences, this represents an average of 36,347 cases per year. The number of cases for $2010(45,155)$ represented $26 \%$ increased compared to cases in 2009, which also showed a $14 \%$ increase compared to 2008 (MPPS 2009, 2010). The highest numbers of malaria cases in Venezuela are currently reported in four states: Bolivar, Amazonas, Delta Amacuro and Sucre. When combined, these states have less than $10 \%$ of the country's total population.

Malaria transmission through blood transfusion constitutes an important public health problem in several regions of the world where the disease is endemic and routine malaria diagnoses are carried out by the microscopic visualization of parasites in thick/thin blood smears. This method is not very sensitive at low parasite densities and it is also inadequate for the examination of a large number of samples or for large-scale epidemiological surveys (Schindler et al. 2001, Kitchen \& Chiodini 2006).

Financial support: CDCH, UCV (PG 09-36-5130), UDO (CI-2-0401021367/07 to MDD and HR)

+ Corresponding author: contrerc@gmail.com

Received 27 June 2010

Accepted 14 January 2011
However, different diagnostic methods exist that are very effective and can be used for epidemiological investigations and the evaluation of malaria control strategies. Methods based on immunofluorescence, immune-haemagglutination, immunoprecipitation and immunoenzymatic [enzyme linked immunosorbent assay (ELISA)] assays have been widely used to detect antibodies against the erythrocytic stages of the parasite (Danis-Lozano et al. 1999).

Two studies have been carried out in blood donors from endemic and non-endemic areas of Venezuela. First, Nuñez et al. (1992) examined the prevalence of Plasmodium falciparum seropositivity in 890 blood donors from different geographical areas. As established by ELISA, the overall prevalence was determined to be $1.7 \%$; however, these seropositive cases were not confirmed. Prevalence was higher in the samples obtained in Sucre $(2.5 \%)$ and Apure (1.7\%), both foci of malaria transmission. In addition, Contreras et al. (1999) studied sera from 1,000 blood donors by immunoflourescent assay (IFA) and IgG-ELISA tests designed using a synchronized in vitrocultured Venezuelan isolate of $P$. falciparum as the antigen source. In Caracas, a non-endemic area, they found $0.8 \%$ of donors positive by the IFA test and by ELISA. In Ciudad Bolívar, an endemic area, they found 3.8\% of donors positive by the IFA test and $2 \%$ positive by ELISA. These studies established that there is a high risk of malaria transmission via blood transfusion, indicating the need for more sensitive diagnostic techniques and preventive measures in the blood banks.

However, the analysis of 15,158 blood samples from donors in France gave a specificity of $99 \%$ for the ELISA 
and $92.6 \%$ concordance with the IFA for the diagnosis of Plasmodium vivax and P. falciparum, showing that applying these techniques for blood bank screening could contribute to the prevention of malaria transmission by blood transfusion (Elghouzzi et al. 2008).

Thus, serological methods constitute a potential diagnosis method for the detection of antimalarial antibodies; furthermore, these methods can be effectively used to screen large numbers of blood samples from donors. This screening allows for the estimation of the risk of transfusion-transmitted malaria. However, here, we studied the presence of malarial antigen and malariaspecific antibodies in samples from blood donors from endemic and non-endemic areas of Venezuela using commercial ELISA kits.

\section{SUBJECTS, MATERIALS AND METHODS}

Blood sample collection - Blood samples from both genders and different age groups were obtained from donors using the recommended biosecurity measures. In total, 762 blood donors were randomly chosen and blood samples were collected between January-July of 2004. Blood was collected from 196 donors from Caracas University Hospital, Capital District, 193 donors from the Antonio Patricio de Alcala, University Hospital in Cumana, Sucre, 187 donors from the Ruiz and Paez Hospital in Ciudad Bolívar, Bolívar, and 186 donors from the Jose Gregorio Hernandez Hospital in Puerto Ayacucho, Amazonas. Caracas is non-endemic for malaria. Sucre is endemic for $P$. vivax. Both Bolívar and Amazonas are endemic for P. falciparum (around 25\%), P. vivax (around 75\%) and less frequently Plasmodium malariae (less than 0.5\%) (MPPS 2010).

In addition, we collected 14 blood samples from patients positively diagnosed for $P$. vivax infection by polymerase chain reaction (PCR) from the village of El Paujil in Sucre, 17 blood samples from patients diagnosed with a Plasmodium infection (8 P. vivax and 9 P. falciparum) from San Fernando de Atabapo, Amazonas, and 25 randomly chosen samples from the village of Cangua, Sucre. Positive samples were used as positive controls during the standardization of the kits and detection of Plasmodium antibodies and $P$. falciparum antigens in blood samples. In addition, we used $P$. falciparum NF54 strain continuous cultures, adjusted to different levels of parasitaemia, as positive controls for the antigen diagnostic kit. All blood bank samples were tested for the presence of HIV-1/2, hepatitis B-C, Toxoplasma gondii, Trypanosoma cruzi and Treponema pallidum by ELISA.

Pan Malaria Antibody kit (PMAb) cELISA - This test detects IgG antibodies against all Plasmodium species. This technique was applied according to the manufacturer's instructions (Cellabs Pty Ltd, Sydney, Australia) and is based on an indirect ELISA, whereby the wells are previously coated with a panel of recombinant antigens from several parasite species. Plasma samples and controls were diluted 1:100. All samples from the endemic populations and the blood banks were assayed twice; samples that tested positive were assayed once more. The values obtained were then averaged for subsequent analysis.
In Venezuela, all donors must be free of symptoms and have been away from active malaria transmission areas for the last six months. Thus, it is expected that all blood donors would have developed an immune response by the time of sampling.

Malaria antigen cELISA - This test is designed as a confirmatory assay for $P$. falciparum infections in blood samples or patients and is used when the results of the diagnosis by traditional microscopic examination are doubtful. This kit contains a $P$. falciparum-specific monoclonal antibody directed against merozoite stage histidine rich protein 2 (PFHRP-II), which can circulate in the blood of infected patients 14 days after parasite death. This technique was applied according to the manufacturer's instructions (Cellabs Pty Ltd, Sydney, Australia) and is based on an indirect ELISA whereby the wells are previously coated with a monoclonal capture antibody specific to PFHRP-II. Briefly, anticoagulated whole blood samples and controls were freeze-thawed three times. All samples from the endemic populations and the blood banks were assayed twice and those that tested positive or had a coefficient of variation higher than $10 \%$ were assayed once more. The values obtained were then averaged for subsequent analysis.

ELISA standardization - The absorbance readings [optical density (OD)] by the cELISA Antigen (CAg) Kit from cultures with different parasitaemias $(0.5,0.05$ and $0.03 \%$ ) of the P. falciparum strain NF54 showed a close fit to the calibration curve. Because the results with $0.05 \%$ parasitaemia were reproducible, showed a consistently high OD and were within the linear scale of the equipment, aliquots of $0.05 \%$ parasitaemia cultures were used as positive controls throughout the study instead of blood from P. falciparum-infected patients. The ODs of the positive control at different dilutions for the PMAb Kit also closely aligned with the calibration curve, except for the 1:200 dilution, which varied slightly from its expected value. Therefore, previously tested blood samples from malaria-infected patients were used as positive controls.

For the calculation of the cut-off values for the CAg and PMAb assays we used the readouts of the samples from the Caracas blood bank (non-endemic area). The average OD value measured for the CAg of these samples was 0.036 with a standard deviation of 0.016 . The average OD value measured for the PMAb using the samples from Caracas was 0.019 , with a standard deviation of 0.016.

We calculated the cut-off value for the CAg according to the manufacturer's instructions as cut-off $=0.1+$ $\mu$, where $\mu$ is the average OD value of the non-endemic samples (Caracas blood bank). The cut-off value for the CAg was then calculated as 0.136 .

In the same way, the cut-off value for the PMAb was calculated as: cut-off $=\mu+3 \sigma$, where $\mu$ is the average OD value of the non-endemic samples and $\sigma$ is the standard deviation of $\mu$. The cut-off value for the PMAb was then calculated as 0.067 .

Molecular diagnosis of malaria - Molecular detection of P. falciparum, P. vivax and P. malariae was carried out using PCR for all of samples studied to verify 
the presence or absence of DNA from these Plasmodium species. As described in Snounou et al. (1993), a nested PCR protocol was used to amplify the $18 \mathrm{~S}$ rRNA genes with primers specific for $P$. falciparum, $P$. vivax and $P$. malariae (Snounou et al. 1993).

Total genomic DNA was extracted from blood samples using the Wizard Genomic DNA Extraction Kit (Promega Corp, Madison, WI, USA) according to the manufacturer's instructions. A volume of $300 \mu \mathrm{L}$ of blood was used and later stored at $-20^{\circ} \mathrm{C}$ until further analysis.

Both amplification rounds of the nested PCR were done in a $20-\mu \mathrm{L}$ volume using Taq polymerase buffer (10 mM Tris- $\mathrm{HCl}, \mathrm{pH} 9.0 ; 50 \mathrm{mM} \mathrm{KCl} ; 0.1 \%$ Triton X-100), $200 \mu \mathrm{M}$ of each dNTP, $0.75 \mathrm{U}$ of Taq polymerase (Promega Corp), $2.0 \mathrm{mM}$ of $\mathrm{MgCl} 2,175 \mathrm{nM}$ of oligonucleotides and $2 \mu \mathrm{L}$ of diluted DNA (about $100 \mathrm{ng}$ ) or $1 \mu \mathrm{L}$ product from the first amplification round. The first and second rounds of amplification were carried out as previously described (Snounou et al. 1993). Amplified products were separated by electrophoresis using a $2 \%$ agarose gel in $1 \mathrm{X}$ Tris- $\mathrm{HCl}, \mathrm{H} 3 \mathrm{BO} 3$ and EDTA buffer (TBE). The gel contained $0.5 \mu \mathrm{g} / \mathrm{mL}$ of ethidium bromide and the DNA was visualized using an ultraviolet transilluminator.

Statistical analysis - Sensitivity, specificity and positive and negative predictive values were calculated for the cELISA antigen using the results of the 59 samples (PCR and microscopy negative, from non-endemic areas) used as negative controls. Fifteen of these samples were later artificially infected with the $P$. falciparum NF54 strain at a parasitaemia of $0.05 \%$ and used as positive controls. Nine P. falciparum-infected samples from Amazonas were also used as malaria Ag-positive controls. The characterization of the PMAb was carried out using the results of the same 59 samples used as negative controls for antigen detection, as well as using 14 samples from El Paujil, two samples from Cangua (positive by PCR to $P$. vivax), Sucre, and 17 samples from Amazonas infected with Plasmodium species as positive controls. Three positive controls from the antibody kits were also used for the calculations. The $95 \%$ confidence intervals for these parameters were determined according to a general method (Newcombe 1998). ANOVA analyses and Student-Newman-Keuls post hoc tests were carried out using the software package SPSS 11.5 (SPSS Inc, Chicago, IL, USA).

As the PCR method was used as the gold standard to determine the sensitivity and specificity of the ELISA kits, it was not possible to determine the sensitivity/ specificity of the PCR method. We have determined the sensitivity/specificity of the PCR method in the same malaria areas in a previous study (Rodulfo et al. 2007a).

Ethics - Previous to the collection and evaluation of human blood samples, patients signed the informed consent form provided, as recommended by the Bioethics and Biosecurity Code of the Science and Technology Ministry and the National Fund for Science and Technology. All procedures for sample collection and evaluation used in this study were approved by the Ethical Committee at the Institute of Immunology, Universidad Central de Venezuela, Caracas, Venezuela.

\section{RESULTS}

The average OD ratios of the negative controls were $0.24 \pm 0.06$ (range 0.10-0.42) for the CAg and $0.40 \pm$ 0.26 (range 0.06-1.12) for the PMAb (Fig. 1). The positive controls for the CAg and PMAb showed average OD ratios of $5.03 \pm 1.51$ (range 2.99-7.84) and $10.72 \pm 4.88$ (range 4.57-19.84), respectively.

When analyzing samples infected with either $P$. vivax (22 samples) or P. falciparum (9 samples), we found eight samples with OD ratios below one for PMAb (Table I). The average OD ratio for PMAb of samples infected with P. falciparum (2.07) was lower than the average OD ratio of the samples infected with $P$. vivax (8.95). However, all $P$. falciparum positive samples showed CAg OD ratios above one, with an average of $8.62 \pm 3.33$ (range 3.27-12.58).
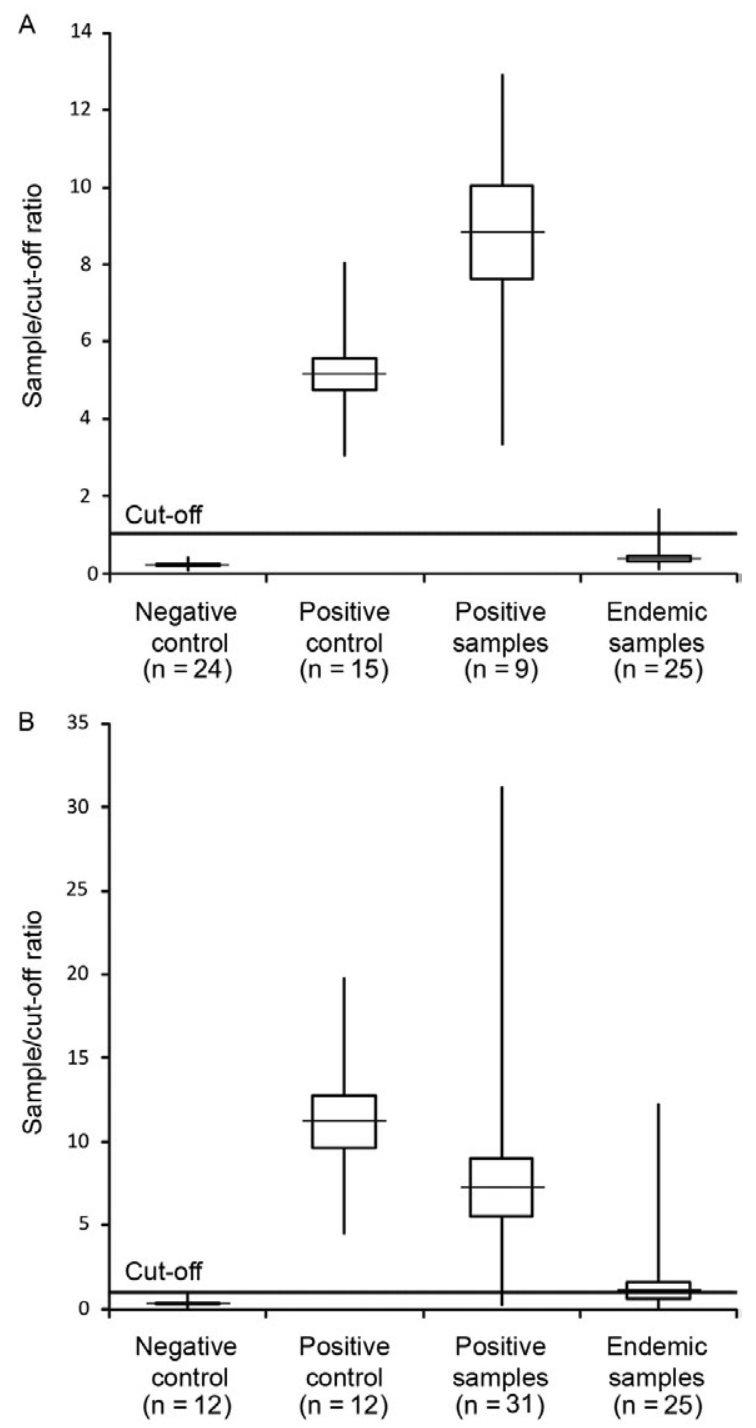

Fig. 1: optical density ratio (sample/cut-off) of the Plasmodium falciparum antigen (A) and antibodies against Plasmodium (B) for the negative and positive controls run in each trial, as well as for blood samples infected with $P$. falciparum and Plasmodium vivax (San Fernando de Atabapo and Cajigal) and the random samples from the population endemic for $P$. vivax used to evaluate the kit. 
On the one hand, one of the samples from Sucre, a $P$. vivax endemic area, gave a CAg OD ratio above one (1.66) (Table II). However, this sample showed a PMAb OD ratio of 0.58 . On the other hand, five of the samples from Sucre $(20 \%)$ were above the cut-off for PMAb. When analyzed by PCR, only two of the four seropositive samples for PMAb were positive for $P$. vivax (showing PMAb OD ratios of 12.27 and 3.70). The other three PMAb seropositive samples were not infected and showed low CAg OD ratios.

Further, we analyzed blood samples from 762 donors from endemic and non-endemic malaria regions by both CAg and PMAb (Fig. 2). CAg analysis of these samples showed only one donor from Caracas (non-endemic area) with an OD ratio above one; however, this sample was negative for all of the tested Plasmodium species when analyzed by PCR. This individual, according to the questionnaire, had not visited any malaria-endemic regions during the previous year. The average OD ratios among the blood banks showed statistical differences (ANOVA, $\mathrm{F}=17.47, \mathrm{p}<0.001$ ), whereby the average CAg OD ratios for Caracas $(0.27 \pm 0.12)$ and Puerto Ayacucho $(0.25 \pm 0.10)$ were lower than Ciudad Bolívar $(0.31$ $\pm 0.17)$ and Cumaná $(0.34 \pm 0.13)$.

\section{TABLE I}

Average results of the absorbance readings [optical density (OD)] ratios for the cELISA Pan Malaria Antibody Kit (PMAb) of Plasmodium infected samples

\begin{tabular}{lcccc}
\hline & & & & Sample/cut-off \\
& & & PMAb & OD ratio \\
\cline { 2 - 5 } Species (PCR) & $\mathrm{n}$ & + & - & $\overline{\mathrm{X}} \pm \mathrm{SD}$ \\
\hline Plasmodium vivax & 22 & 18 & 4 & $8.95 \pm 9.87$ \\
Plasmodium falciparum & 9 & 5 & 4 & $2.07 \pm 3.09$ \\
\hline
\end{tabular}

PCR: polymerase chain reaction; SD: standard deviation.

\section{TABLE II}

Results of the absorbance readings optical density ratios for the cELISA Antigen (CAg) and Pan Malaria Antibody Kit (PMAb) for the Sucre endemic samples that showed ratios above the cut-off with either kit

\begin{tabular}{|c|c|c|c|}
\hline Sample & $\begin{array}{c}\text { PCR } \\
\text { Plasmodium vivax/ } \\
\text { Plasmodium falciparum }\end{array}$ & PMAb & $\mathrm{CAg}$ \\
\hline 2601 & - & 0.58 & 1.66 \\
\hline 2605 & - & 1.22 & 0.28 \\
\hline 2802 & - & 1.05 & 0.23 \\
\hline 2901 & - & 1.78 & 0.74 \\
\hline 3007 & + & 12.27 & 0.18 \\
\hline 3909 & + & 3.70 & 0.56 \\
\hline
\end{tabular}

PCR: polymerase chain reaction.
When analyzed by PMAb (Fig. 2B), two samples from Caracas, three from Ciudad Bolívar, six from Puerto Ayacucho and seven from Cumaná had OD ratios above one. However, when analyzed by PCR, none of these samples proved to be infected with any of the tested Plasmodium species. These individuals, according to their questionnaires, had not travelled to active malaria transmission areas during the previous year. The average OD ratio among the blood banks showed statistical differences (ANOVA, $F=91.83, \mathrm{p}<0.001$ ) and the OD ratio for the samples from Caracas was the lowest $(0.28$ $\pm 0.23)$ followed by Cumaná $(0.49 \pm 0.29)$, Ciudad Bolí$\operatorname{var}(0.60 \pm 0.15)$ and Puerto Ayacucho $(0.66 \pm 0.26)$. The prevalence of antibodies against the malaria antigens detected by PMAb was 1.02, 1.60, 3.23 and 3.63\% for Caracas, Ciudad Bolívar, Puerto Ayacucho and Cumaná blood banks, respectively. The prevalence of antibodies in the endemic Sucre population was $20 \%$.
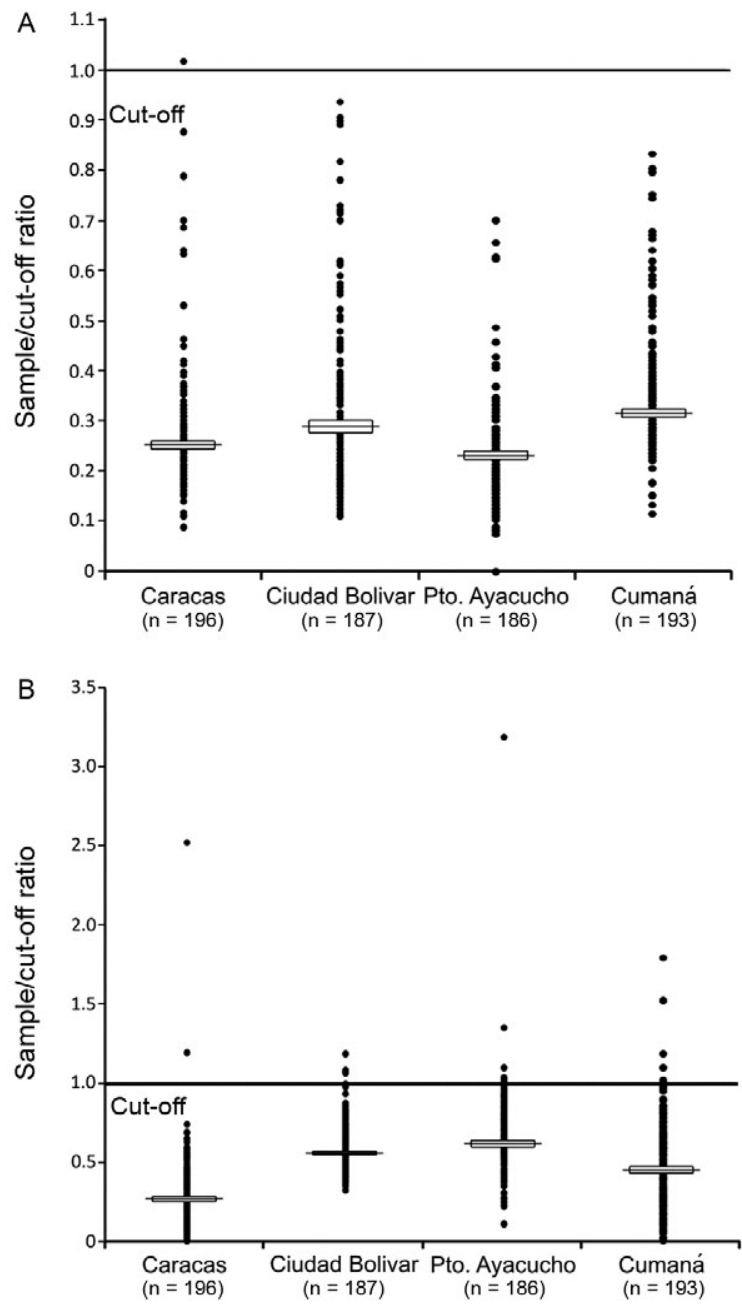

Fig. 2: optical density ratio (sample/cut-off) of the Plasmodium falciparum antigen (A) and antibodies against Plasmodium (B) of the samples from the blood banks used in the study. Individuals from Caracas are the only ones not close to a malaria endemic area, individuals from Ciudad Bolívar and Puerto Ayacucho live close to an endemic area for P. falciparum and Plasmodium vivax and individuals from Cumaná live close to an endemic area for $P$. vivax. 
TABLE III

Evaluation of the cELISA Antigen (CAg) and Pan Malaria Antibody Kit (PMAb) as methods for malaria diagnosis

\begin{tabular}{lcccc}
\hline & $\begin{array}{c}\text { Sensitivity } \\
\%(95 \% \mathrm{CI})\end{array}$ & $\begin{array}{c}\text { Specificity } \\
\%(95 \% \mathrm{CI})\end{array}$ & $\begin{array}{c}\text { PPV } \\
\%(95 \% \mathrm{CI})\end{array}$ & $\begin{array}{c}\text { NPV } \\
\%(95 \% \mathrm{CI})\end{array}$ \\
\hline $\mathrm{CAg}$ & $100(82.83-100)$ & $99.75(99.01-99.96)$ & $92.31(73.40-98.66)$ & $100(99.41-100)$ \\
$\mathrm{PMAb}$ & $82.22(67.42-91.49)$ & $97.37(95.93-98.32)$ & $63.79(50.06-75.69)$ & $98.98(97.92-99.52)$ \\
\hline
\end{tabular}

CI: confidence interval; NPV: negative predictive value; PPV: positive predictive value.

We found high values of sensitivity, specificity, positive predictive value (PPV) and negative predictive value (NPV) when CAg was used as a diagnostic method for the detection of $P$. falciparum (Table III). However, although PMAb had a high specificity and NPV, it showed a lower sensitivity and PPV for the diagnosis of all Plasmodium species.

\section{DISCUSSION}

The elevated malaria indexes found in endemic areas of Venezuela increase the risk of blood transfusion transmission. Our results showed that there is some risk of this type of transmission, with antibody seroprevalences ranging between $1-3.6 \%$ in the endemic states studied. Although no infected samples were detected by PCR, seroprevalences should be zero if the screening measures were $100 \%$ efficient. Our results are similar to a previous analysis of 1,000 blood donors from non-endemic (Caracas) and endemic (Bolívar) areas of Venezuela. This study showed seropositivities of $0.8 \%$ and $3.8 \%$, respectively, using IFA and ELISAs to detect $P$. falciparumspecific IgG antibodies (Contreras et al. 1999). Because values from this report were for $P$. falciparum-specific antibodies only, our findings demonstrate a decrease in the seroprevalence for Bolívar; the $1.60 \%$ prevalence in our study included antibodies against $P$. vivax, $P$. falciparum and $P$. malariae. However, the high seroprevalence found in samples from the endemic community in Sucre shows that transfusion transmission risk is much higher in areas of active transmission.

The prolonged contact of individuals to malaria parasites has been reported to decrease parasite densities and host symptoms; further, there are reports of infected asymptomatic patients in endemic areas of Venezuela that were not properly diagnosed by microscopic examination (Postigo et al. 1998, Rodulfo et al. 2007b). In the Brazilian Amazon, a PCR analysis of 400 samples collected from four blood transfusion centres, found frequencies of malaria-infected blood donors ranging from 1-3\% (Fugikaha et al. 2007). Of the infected donors, nine were non-symptomatic positive individuals infected with both $P$. vivax and $P$. falciparum. These results highlight the risks of transfusion-transmitted malaria in endemic areas.

In Venezuela, even though malaria is very prevalent, there is no consensus in the measures taken to prevent transfusion-transmitted malaria; currently, only a questionnaire for the deferral of high-risk individuals is used. Diop et al. (2009), who compared various screen- ing strategies, concluded that the medical questionnaire is not efficient for the deferral of asymptomatic carriers. Instead, they propose to introduce Plasmodium screening methods to increase efficiency.

The screening of blood bank donors in endemic areas is of higher importance because of the two opposing factors in play: the scarce number of blood donors and the increased risk of disease transmission. Interestingly, PCR analysis of 115 individuals, rejected as potential donors because they had travelled to or lived in malaria-endemic areas within the previous year, showed that none were infected with any of the Plasmodium species (Castillo \& Ramírez 2005). Thus, efficient diagnostic tests for blood donor screening need to be implemented in order to avoid losing valuable blood donors.

In our study, the CAg and PMAb tests showed high sensitivities (100\% and $82.2 \%$, respectively) and specificities $(99.8 \%$ and $97.4 \%$ ), thus proving to be useful for the screening of donor blood samples to detect transmission risk, especially in endemic areas. However, varying results have been found in other studies using PMAb and CAg ELISA-based tests. For instance, in a study using cELISA and ICT Malaria Pf diagnostic kits to detect malaria, the cELISA kit had a sensitivity of $71 \%$ for 203 patients infected with $P$. falciparum (Silvie et al. 2002). However, for samples from travellers living in Utah, USA, the cELISA PMAb test exhibited better overall sensitivity $(95.5 \%)$ and specificity $(92.2 \%)$ compared to the Newmarket Malaria ELISA $(68.1 \%$ and $96.1 \%$, respectively) and IFA tests ( $86.4 \%$ and $91.7 \%$, respectively) (She et al. 2007). Consistent with this finding, the overall sensitivity and specificity of the cELISA P. falciparum antigen detection assay was $98.8 \%$ and $100 \%$, when used on samples from the Thailand-Myanmar border (Noedl et al. 2006); importantly, sensitivity did not decrease at a low parasite density. However, the characterization of the cELISA method was not complete because Noedl's group only examined symptomatic outpatients.

Many studies using ELISA tests with different protein targets have also shown good results compared to other diagnostic tests. For example, the IFA test showed a specificity of $99 \%$ and a sensitivity of $80 \%$, while the IgG-ELISA gave a specificity of $98 \%$ and a sensitivity of $68 \%$ for samples from Venezuela (Contreras et al. 1999). In addition, in the analysis of 200 P. vivax-infected patients and 507 healthy blood bank donors, ELISAs detecting IgG and IgM antibodies directed against a recombinant transmembrane region of the merozoite surface 
protein 1 (MSP1) exhibited a sensitivity of $99.5 \%$ with 99.4\% specificity (Lim et al. 2002). Similarly, using the same protein to capture IgM antibodies against $P$. vivax, Park et al. (2008) showed high levels of sensitivity and specificity $(97.7 \%$ and $99.1 \%$, respectively) even at low levels of parasitaemia.

The high sensitivity and specificity values found for the CAg test in our study (all infected samples showed high OD ratios) confirms the usefulness of HRP-II as a valuable marker for $P$. falciparum infections. However, its utility in Venezuelan blood bank screening is limited as this technique cannot detect $P$. vivax antigens and $75 \%$ of the malaria cases in Venezuela are caused by $P$. vivax (MPPS 2010).

Antibodies have been shown to be very effective for the detection of acute and/or recent infections and are valuable tools for screening blood donors to detect their risk for malaria transmission. In an Australia study detecting antibodies by ELISA, Seed et al. (2005) found that a targeted screening strategy, combined with a sensitive antibody screening test, increased the efficacy of screening when used in a four-month cellular component restriction period for donors with a declared malarial risk.

The period of time it takes infected individuals to become seronegative by ELISA tests after being cured depends on factors such as the patient's immunological status, the target antigens and the patient's history of infection (Pinto et al. 2001). For example, antibody levels significantly decreased two months after $P$. vivax infection in 32 individuals from an endemic area of Brazil (Soares et al. 1999). In addition, a study evaluating antibody levels against seven different antigens showed that antibodies produced in response to a primary infection had a reduced half-life response compared to antibodies generated after a second exposure to antigen. Moreover, individuals infected with $P$. vivax who showed negative results for antibodies against pvMSP1, were positive for other recombinant proteins from this species (Galinski \& Barnwell 1996). However, examination of individuals that had been briefly exposed to malaria in endemic zones of Brazil, using a recombinant $P$. vivax apical membrane merozoite antigen, showed that antibodies against this antigen persisted for at least seven years after infection, even when the individuals were not subjected to further exposure (Morais et al. 2006). These reports demonstrate that the levels of each antigen-specific antibody are independent of each other; thus, when using an antibody screening method, the target population must be characterized to establish the antibody's efficiency as a risk assessment method.

Evaluation of 10,615 blood donor samples collected from eight blood banks in France with the Diamed ELISA malaria antibody test, which detects $P$. vivax and $P$. falciparum-specific $\operatorname{IgG}$ and $\operatorname{IgM}$ antibodies, showed positive frequencies ranging from $1.83-4.39 \%$ (Elghouzzi et al. 2008). These results were similar to those found in a study of 1,756 blood donor samples from endemic and non-endemic areas of Saudi Arabia, where a $9.1 \%$ antibody-positive rate and $0.18 \%$ antigen prevalence were found for the samples from the endemic region when analyzed by ELISA (Saeed et al. 2002). In the non-endemic region, $4.8 \%$ antibody and $0.15 \%$ antigen positive rates were found. These results suggest that serological tests could be used to confirm the absence of infection in donor samples, thus avoiding discarding usable blood donors from endemic areas.

Serological tests for the detection of infected individuals based on $\operatorname{IgG}$ antibodies are less useful in hyperendemic areas, as the presence of antibodies bears no relation to the presence or absence of the parasite in these cases. For example, examination of $511 \mathrm{Am}-$ erindians inhabiting the northern Venezuelan Amazon showed that $69.6 \%$ of individuals from two-five years of age were positive for antibodies against $P$. falciparum antigens and $96.9 \%$ of the individuals from $31-40$ years of age tested positive (Sanchez et al. 1990). Antibody prevalences of over $90 \%$ have also been found in the states of Amazonas and Sucre in population-based studies (Sanchez et al. 1990, Mundaray 2002).

Our results confirm that a risk of blood transfusiontransmitted malaria exists in blood banks in the malaria endemic areas of Venezuela. We recommend the use of medical and epidemiological questionnaires in nonendemic areas. In individuals who had lived in those areas for a long time, a deferral time of six months can be used when travelling to endemic areas. However, either malaria screening or longer deferral times should be applied to individuals who had lived in endemic areas for a considerable period of time. In contrast, in endemic areas the use of appropriate diagnostic techniques needs to be implemented. Currently, diagnostic tests are only carried out at the Puerto Ayacucho blood bank, where malaria diagnosis is performed by microscopic examination. In addition to the questionnaire, we recommend the use of an antibody screening method in conjunction to the microscopic detection of parasites with Giemsa or fluorescence staining or another alternative method with proven high sensitivity and specificity values. These methods would help to avoid discarding valuable blood donations that are much needed in endemic areas.

\section{ACKNOWLEDGEMENTS}

To Dr GR Rajasekariah, from Cellabs Pty Ltd, for the donation of the Pan Malaria Antibody and the Malaria Antigen kits, to Dr Alexis Rodriguez-Acosta, from the Tropical Medicine Institute, Universidad Central de Venezuela, and Dr Mariolga Berrizbeitia, from the Universidad de Oriente, for the critical revision of the manuscript.

\section{REFERENCES}

Castillo CM, Ramírez C 2005. Malaria screening in blood donors in Cali, Colombia. Biomedica 25: 203-210.

Contreras CE, Pance A, Marcano N, González N, Bianco N 1999. Detection of specific antibodies to Plasmodium falciparum in blood bank donors from malaria-endemic and non-endemic areas of Venezuela. Am J Trop Med Hyg 60: 948-953.

Danis-Lozano R, Rodriguez MH, Gonzalez-Ceron L, Hernandez-Avila M 1999. Risk factors for Plasmodium vivax infection in the Lacandon forest, southern Mexico. Epidemiol Infect 122: 461-469.

Diop S, Ndiaye M, Seck M, Chevalier B, Jambou R, Sarr A, Dièye TN, Touré AO, Thiam D, Diakhaté L 2009. Prevention of transfusion transmitted malaria in endemic area. Transfus Clin Biol 16: 454-459. 
Elghouzzi MH, Senegas A, Steinmetz T, Guntz P, Barlet V, Assal A, Gallian P, Volle P, Chuteau C, Beolet M, Berrebi S, Filisetti D, Doderer C, Abdelrahman T, Candolfi E 2008. Multicentric evaluation of the DiaMed enzyme-linked immunosorbent assay malaria antibody test for screening of blood donors for malaria. Vox Sang 94: 33-40.

Fugikaha E, Fornazari PA, Penhalbel R de S, Lorenzetti A, Maroso RD, Amoras JT, Saraiva AS, Silva RU, Bonini-Domingos CR, Mattos LC, Rossit AR, Cavasini CE, Machado RL 2007. Molecular screening of Plasmodium sp. asymptomatic carriers among transfusion centers from Brazilian Amazon region. Rev Inst Med Trop Sao Paulo 49: 1-4.

Galinski MR, Barnwell JW 1996. Plasmodium vivax: merozoites, invasion of reticulocytes and considerations for malaria vaccine development. Parasitol Today 12: 20-29.

Kitchen AD, Chiodini PL 2006. Malaria and blood transfusion. Vox Sang 90: 77-84.

Lim KJ, Park JW, Sohn MJ, Lee S, Oh JH, Kim HC, Bahk YY, Kim YS 2002. A direct sandwich ELISA to detect antibodies against the C-terminal region of merozoite surface protein 1 could be a useful diagnostic method to identify Plasmodium vivax exposed persons. Parasitol Res 88: 855-860.

Morais CG, Soares IS, Carvalho LH, Fontes CJ, Krettli AU, Braga EM 2006. Antibodies to Plasmodium vivax apical membrane antigen 1: persistence and correlation with malaria transmission intensity. Am J Trop Med Hyg 75: 582-587.

MPPS - Ministerio del Poder Popular para la Salud 2009. Boletin epidemiologico semanal. Año LVI, Semana Epidemiológica 52 (Accessed September 15, 2010). Available from: www.mpps.gob.ve/ modules.php?name=Downloads.

MPPS - Ministerio del Poder Popular para la Salud 2010. Boletin epidemiologico semanal. Año LVII, Semana Epidemiológica 40 (Accessed October, 2010). Available from: www.mpps.gob.ve/ modules. php? name $=$ Downloads .

Mundaray N 2002. Prevalencia de anticuerpos a Plasmodium vivax en la población de Pozo Colorado, Edo, Sucre, Thesis, Universidad de Oriente, Cumaná, 33 pp.

Newcombe RG 1998. Two-sided confidence intervals for the single proportion: comparison of seven methods. Stat Med 17: 857-872.

Noedl H, Yingyuen K, Laoboonchai A, Fukuda M, Sirichaisinthop J, Miller RS 2006. Sensitivity and specificity of an antigen detection ELISA for malaria diagnosis. Am J Trop Med Hyg 75: 1205-1208.

Nuñez L, Linares J, Perez AH 1992. Seroprevalencia de anticuerpos a Plasmodium falciparum en donantes voluntarios de varias ciudades de Venezuela. Sangre (Barc) 37: 141-143.

Park JW, Yoo SB, Oh JH, Yeom JS, Lee YH, Bahk YY, Kim YS, Lim KJ 2008. Diagnosis of vivax malaria using an IgM capture ELISA is a sensitive method, even for low levels of parasitemia. Parasitol Res 103: 625-631.
Pinto AYN, da Silva Ventura AMR, de Souza JM 2001. IgG antibody response against Plasmodium vivax in children exposed to malaria before and after specific treatment. J Pediatr 77: 299-306.

Postigo M, Mendoza-León A, Pérez HA 1998. Malaria diagnosis by the polymerase chain reaction: a field study in south-eastern Venezuela. Trans $R$ Soc Trop Med Hyg 92: 509-511.

Rodulfo H, De Donato M, Mora R, González L, Contreras CE 2007a. Comparison of the diagnosis of malaria by microscopy, immunochromatography and PCR in endemic areas of Venezuela. Braz J Med Biol Res 40: 535-543.

Rodulfo H, de Donato M, Quijada I, Peña A 2007b. High prevalence of malaria infection in Amazonas state, Venezuela. Rev Inst Med Trop Sao Paulo 49: 79-85.

Saeed AA, Al Rasheed AM, Al Nasser I, Al Onaizi M, Al Kahtani S, Dubois L 2002. Malaria screening of blood donors in Saudi Arabia. Ann Saudi Med 22: 329-332.

Sanchez E, Perez HA, Martinez C 1990. Malaria in the Amazon. Prevalence of Plasmodium falciparum antibodies in Amerindians inhabiting the Venezuelan Amazon. Ann Trop Med Parasitol 84: 307-312.

Schindler HC, Montenegro L, Montenegro R, Carvalho AB, Abath FG, Jaureguiberry G 2001. Development and optimization of polymerase chain reaction-based malaria diagnostic methods and their comparison with quantitative buffy coat assay. Am J Trop Med Hyg 65: 355-361.

Seed CR, Kitchen A, Davis TM 2005. The current status and potential role of laboratory testing to prevent transfusion-transmitted malaria. Transfus Med Rev 19: 229-240.

She RC, Rawlins ML, Mohl R, Perkins SL, Hill HR, Litwin CM 2007. Comparison of immunofluorescence antibody testing and two enzyme immunoassays in the serologic diagnosis of malaria. J Travel Med 14: 105-111.

Silvie O, Thellier M, Rosenheim M, Datry A, Lavigne P, Danis M, Mazier D 2002. Potential value of Plasmodium falciparum-associated antigen and antibody detection for screening of blood donors to prevent transfusion-transmitted malaria. Transfusion 42: $357-362$.

Snounou G, Viriyakosol S, Zhu XP, Jarra W, Pinhero L, do Rosario VE, Thaithong S, Brown KN 1993. High sensitivity of detection of human malaria parasites by the use of nested polymerase chain reaction. Mol Biochem Parasitol 61: 315-320.

Soares IS, da Cunha MG, Silva MN, Souza JM, Del Portillo HA, Rodrigues MM 1999. Longevity of naturally acquired antibody responses to the $\mathrm{N}$ - and $\mathrm{C}$-terminal regions of Plasmodium vivax merozoite surface protein 1. Am J Trop Med Hyg 60: 357-363.

WHO - World Health Organization 2008. World malaria report 2008, WHO Press, Geneva, 215 pp. 